Proceedings of the 15th Czech and Slovak Conference on Magnetism, Košice, Slovakia, June 17-21 2013

\title{
Ground-State Phase Diagram of the Extended Ising Model on the Shastry-Sutherland Lattice
}

\author{
H. ČENČARIKOVÁ ${ }^{a, *}$, P. FARKAŠOVSKÝ ${ }^{a}$ AND P. PUChalA ${ }^{b}$ \\ ${ }^{a}$ Institute of Experimental Physics SAS, Watsonova 47, 04001 Košice, Slovakia \\ ${ }^{b}$ Institute of Physics, Faculty of Sciences, P. J. Šafarik University, Park Angelinum 9, 04154 Košice, Slovakia \\ The ground-state phase diagram of the Ising model on the Shastry-Sutherland lattice with the first $\left(J_{1}\right)$, second \\ $\left(J_{2}\right)$, third $\left(J_{3}\right)$ and fourth $\left(J_{4}\right)$ nearest neighbour spin couplings is studied using the exact and well controlled \\ numerical method. It is shown that switching on of the $J_{3}$ and $J_{4}$ interactions (in addition to usually considered \\ $J_{1}$ and $J_{2}$ interactions) leads to stabilization of the following dominant magnetic phases with $\mathrm{m} / \mathrm{m}_{\mathrm{s}}=1 / 9,1 / 6$, \\ $4 / 9,1 / 2$ and $5 / 9$, which is manifested in appearance of new magnetization plateaus on the magnetization curve \\ of the model at given values of $m / m_{s}$. The obtained results are consistent with experimental measurements of \\ magnetization curves in selected rare-earth tetraborides.
}

DOI: $10.12693 /$ APhysPolA.126.46

PACS: 75.10.-b, 75.60.Ej, 75.40.Mg

A spin system is frustrated when all local interactions between spin pairs cannot be satisfied at the same time. Frustration can arise from competing interactions or/and from a particular geometry of the lattice, as seen in the triangular lattice. The Shastry-Sutherland lattice (SSL) was considered more than 20 years ago by Shastry and Sutherland [1] as an interesting example of a frustrated quantum spin system with an exact ground state. It can be described as a square lattice with antiferromagnetic (AF) couplings $J_{1}$ between nearest neighbours and additional AF couplings $J_{2}$ between next-nearest neighbours in every second square. This lattice attracted much attention after its experimental realization in the $\mathrm{SrCu}_{2}\left(\mathrm{BO}_{3}\right)_{2}$ compound [2]. The observation of a fascinating sequence of magnetization plateaus $\left(\mathrm{m} / \mathrm{m}_{\mathrm{s}}=1 / 2\right.$, $1 / 3,1 / 4$ and $1 / 8$ ) in this material stimulated futher theoretical and experimental studies of the SSL. As another realization of the SSL the rare-earth tetraborid $\mathrm{TmB}_{4}$ has recently been studied in finite magnetic fields [3]. It was found that the magnetization diagram of $\mathrm{TmB}_{4}$ consists of magnetization plateaus (MPs) located at small fractional values of $m / m_{s}=1 / 7,1 / 8,1 / 9, \ldots$ of the saturation magnetization, followed by the major MP located at $m / m_{s}=1 / 2$. Because of strong crystal field effects, the effective spin model for $\mathrm{TmB}_{4}$ has been suggested to be described by the spin-1/2 Shastry-Sutherland model under strong Ising (or easy-axis) anisotropy [3]

$$
H=J_{1} \sum_{\langle i j\rangle} S_{i}^{z} S_{j}^{z}+J_{2} \sum_{\langle\langle i j\rangle\rangle} S_{i}^{z} S_{j}^{z}-h \sum_{i} S_{i}^{z},
$$

where $S_{i}^{z}= \pm 1 / 2$ denotes the $z$-component of a spin- $1 / 2$ degree of freedom on site $i$ of a square lattice and $J_{1}$, $J_{2}$ are the AF exchange couplings between all nearest neighbour bonds $\left(J_{1}\right)$ and next-nearest neighbour bonds in every second square $\left(J_{2}\right)$. It should be noted that a similar behavior as for $\mathrm{TmB}_{4}$ has been also observed for

\footnotetext{
*corresponding author; e-mail: hcencar@saske.sk
}

other rare-earth tetraborides. For example, for $\mathrm{ErB}_{4}$ the MP has been found at $m / m_{s}=1 / 2[4,5]$, for $\mathrm{TbB}_{4}$ at $m / m_{s}=1 / 2,4 / 9,1 / 3,2 / 9$ and $7 / 9[6]$ and for $\mathrm{HoB}_{4}$ at $\mathrm{m} / \mathrm{m}_{\mathrm{s}}=1 / 3,4 / 9$ and $3 / 5[5]$. The model (1) has been solved recently by numerical $[7,8]$ as well as analytical methods [9] with a conclusion that only the $1 / 3$ plateau is stabilized by $J_{1}$ and $J_{2}$ interactions. The subsequent analytical studies [10] of the model extended by an additional interaction $\left(J_{3}\right)$ along the diagonals of "empty" squares showed that this interaction gives rise to a new MP at one-half of the saturation magnetization. This result and similar ones obtained on the spin- $1 / 2 \mathrm{XXZ}$ model [11] pointed to the fact that the long-range interaction could play the crucial role in stabilization of different MPs.

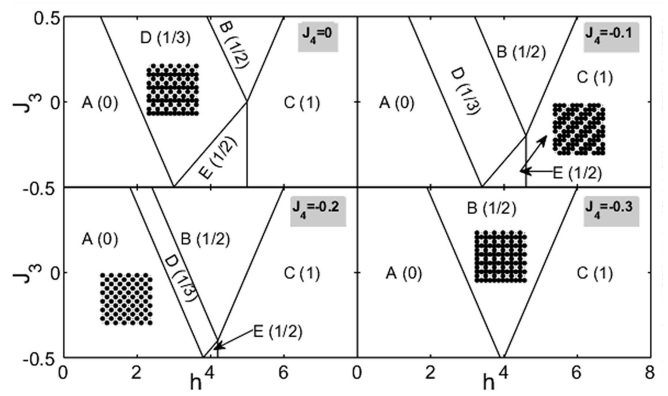

Fig. 1. The $J_{3}-h$ ground-state phase diagrams of the extended Ising model on the SSL for $J_{4} \leq 0$ and $L=$ $12 \times 12$. The numbers in brackets correspond to $\mathrm{m} / \mathrm{m}_{\mathrm{s}}$. Insets: the ground-state spin configurations. The large (small) dots represent the up (down) spin orientation.

For this reason we have performed exhaustive studies of the ground-state phase diagram of the model (1) extended by $J_{3}$ as well as $J_{4}$ interaction (the next-nearest neighbours along edges) by a combination of the Monte Carlo method and our approximate method based on the reduction of the total energy of the system [12]. The results of numerical calculations obtained on $12 \times 12$ cluster with the periodic boundary conditions are summarized in Fig. 1 and Fig. 2 in the form of ground-state phase diagrams in the $J_{3}-h$ plane for different values of $J_{4}$ 


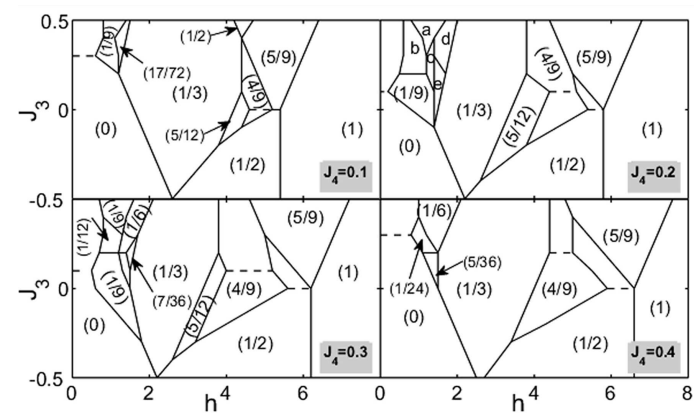

Fig. 2. The $J_{3}-h$ ground-state phase diagrams of the extended Ising model on the SSL for $J_{4}>0$ and $L=12 \times 12$. The numbers in brackets correspond to $\mathrm{m} / \mathrm{m}_{\mathrm{s}}$. Phases $a, b, c, d, e$ correspond to the fractional magnetizations $1 / 9,1 / 12,17 / 72,7 / 36,1 / 4$. The dashed lines represent the phase boundaries between different spin orderings corresponding to the same $\mathrm{m} / \mathrm{m}_{\mathrm{s}}$.

interaction $\left(J_{1}=J_{2}=1\right)$. Let us first discuss the case of $J_{4} \leq 0$ (Fig. 2). For $J_{4}=0$, one can see that the point $J_{3}=0$, corresponding to the ordinary Ising model on the SSL, is the special point of the $J_{3}-h$ phase diagram with the only one intermediate plateau at $m / m_{s}=1 / 3$, in accordance with previous numerical $[7,8]$ as well as analytical [9] results. As soon as $J_{3}$ is nonzero the new MP at $m / m_{s}=1 / 2$ is developed for both $J_{3}$, positive and negative. However, it should be noted that the spin ordering, leading to the total $\mathrm{m} / \mathrm{m}_{\mathrm{s}}=1 / 2$ magnetization is fully different in the regions of positive (the phase $B$ ) and negative (the phase $E$ ) values of $J_{3}$.



Fig. 3. The magnetization curves as functions of $h$ for $J_{4}=0$, different values of and different clusters obtained on the extrapolated set of ground-state configurations.

For $J_{3}>0$ the ground-state spin ordering is formed by axial AF and ferromagnetic lines, while for $J_{3}<0$ the antiparallel diagonal ordering of ferromagnetic bands and lines minimizes the ground-state energy. In both phases, the increasing $\left|J_{3}\right|$ stabilizes the $\mathrm{m} / \mathrm{m}_{\mathrm{s}}=1 / 2$ plateau against the $m / m_{s}=1 / 3$ plateau (the $D$ phase). With increasing $\left|J_{4}\right|$ the $B$ phase is further stabilized while the $E$ and $D$ phases are gradually suppressed and they completely disappear at $J_{4} \sim-0.25$. Below this value, the structure of the phase diagram does not depend on the value of $J_{4}$ and the only intermediate MP is one at $m / m_{s}=1 / 2$. To minimize the finite-size effects on these results we have performed the same calculations on much larger cluster of $24 \times 24$ sites and found that all fundamental features, including the phase boundaries, hold practically unchanged. This indicates that the phase di- agrams as well as the corresponding picture of magnetization processes found on small clusters can be satisfactorily extrapolated on much larger clusters. This is demonstrated in Fig. 3, where the magnetization curves obtained on the extrapolated set of configurations forming the ground-state phase diagrams of the model up to $L=24 \times 24$ are plotted for selected values of $J_{3}$ and $J_{4}$ and practically no finite size effects are observed.

The situation in the opposite limit of $J_{4}>0$ is more complex as illustrate the phase diagrams in Fig. 2 obtained for the $L=12 \times 12$ cluster. Now the phase diagrams exhibit a very rich spectrum of numerical solutions, where in addition to the above mentioned $\mathrm{m} / \mathrm{m}_{\mathrm{s}}=$ $1 / 2$ and $m / m_{s}=1 / 3$ plateaus/phases one can find large domains corresponding to the $m / m_{s}=1 / 6,1 / 9,5 / 12$, $4 / 9,5 / 9$ phases. The same numerical calculations that we have performed on the $L=24 \times 24$ cluster showed that these phases form the basic structure of the phase diagrams also on much larger clusters although their phase boundaries are slightly modified now.

Thus we can conclude that in addition to the previously described $m / m_{s}=1 / 2$ and $m / m_{s}=1 / 3$ plateaus [7-10], the extended Ising model on the SSL with $J_{3}$ and $J_{4}$ interactions can explain the existence of further relevant MPs with $m / m_{s}=1 / 6,1 / 9,5 / 12,4 / 9,5 / 9$ in accordance with experimental observations in rare-earth tetraborides $\left(\mathrm{TmB}_{4}, \mathrm{TbB}_{4}, \mathrm{HoB}_{4}, \mathrm{EuB}_{4}\right)$.

\section{Acknowledgments}

This work was supported by the VEGA grant No.2/0077/13, the APVV grant No. APVV-0097-12 and the ERDF EU grant No. ITMS.26220120005.

\section{References}

[1] B.S. Shastry, B. Sutherland, Physica B and C 108, 1069 (1981).

[2] H. Kageyama, K. Yoshimura, R. Stern, N.V. Mushnikov, K. Onizuka, M. Kato, K. Kosuge, C.P. Slichter, T. Goto, Y. Ueda, Phys. Rev. Lett. 82, 3168 (1999); K. Kodama, M. Takigawa, M. Horvatic, C. Berthier, H. Kageyama, Y. Ueda, S. Miyahara, F. Becca, F. Mila, Science 298395 (2002).

[3] K. Siemensmeyer, E. Wulf, H.-J. Mikeska, K. Flachbart, S. Gabáni, S. Mat'aš, P. Priputen, A. Efdokimova, N. Shitsevalova, Phys. Rev. Lett. 101, 177201 (2008).

[4] S. Michimurea, A. Shigekawa, F. Iga, M. Sera, T. Takabatake, K. Ohoyama, Y. Okabe, Physica B 378380, 596 (2006).

[5] S. Mat’aš, K. Siemensmeyer, E. Wheeler, E. Wulf, R. Beyer,Th. Hermannsdörfer, O. Ignatchik, M. Uhlarz, K. Flachbart, S. Gabáni, P. Priputen, A. Efdokimova, N. Shitsevalova, J. Phys.: Conf. Ser. 200, 032041 (2010).

[6] S. Yoshii, T. Yamamoto, M. Hagiwara, S. Michimura, A. Shigekawa, F. Iga, T. Takabatake, K. Kindo, Phys. Rev. Lett. 101, 087202 (2008).

[7] Z.Y. Meng, S. Wessel, Phys. Rev. B 78, 224416 (2008).

[8] M.C. Chang, M.F. Yang, Phys. Rev. B 79, 104411 (2009). 